\title{
FOURIER SERIES OF FUNCTIONS WITH INFINITE DISCONTINUITIES
}

\author{
BRANKO SARIĆ
}

\begin{abstract}
Using the total $\mathrm{H}_{1}$-integrability concept we shall show that functions, which take on infinite values in the interval $(-\pi, \pi)$ at only finitely many places, can be expanded into a Fourier series over this interval.
\end{abstract}

\section{INTRODUCTION}

As is well-known, significant progress in Fourier analysis has gone hand in hand with progress in theories of integration, $[4,11]$. Perhaps this can be best exemplified by using the so-called total value of the generalized Riemann integrals introduced by Saric in his works [6, 7, 8, 9]. This brand new theory of integration, which takes the notion of residues of real valued functions into account, gives us the opportunity to integrate real valued functions that are not integrable in any of the known integration methods until now. Accordingly, in the main part of this paper, we shall see that realvalued functions, with infinite discontinuities within the interval $(-\pi, \pi)$, can be expanded into a Fourier series over $[-\pi, \pi]$.

\section{Preliminaries}

The Lebesgue measure on the set of all real numbers $\mathbb{R}$ is denoted by $\mu$, however, for $E \subset \mathbb{R}$ we write $|E|$ instead of $\mu(E)$. By $\mathbb{N}$ we denote the set of natural numbers. Given the compact interval $[-\pi, \pi]$ let the collection $\mathcal{I}([-\pi, \pi])$ be a family of all compact subintervals $I$ of $[-\pi, \pi]$. Any real valued function defined on $\mathcal{I}([-\pi, \pi])$ is an interval function. For $f:[-\pi, \pi] \mapsto \mathbb{R}$ the associated interval function of $f$ is an interval function $f: \mathcal{I}([-\pi, \pi]) \mapsto \mathbb{R}$, again denoted by $f,[10]$. A partition $P[-\pi, \pi]$ of $[-\pi, \pi]$ is a finite set (collection) of interval-point pairs $\left(\left[a_{i}, b_{i}\right], x_{i}\right), i=1, \ldots, \nu$,

2010 Mathematics Subject Classification. Primary: 42A24; Secondary: 30B10.

Key words and phrases. total $\mathrm{H}_{1}$ - integrability, Fourier series.

The author's research is supported by the Ministry of Science, Technology and Development, Republic of Serbia (Project ON 174024). 
such that the subintervals $\left[a_{i}, b_{i}\right]$ are non-overlapping $\left(\left(a_{i}, b_{i}\right) \cap\left(a_{j}, b_{j}\right)=\emptyset\right.$ for $i \neq j$, where $\left(a_{i}, b_{i}\right)$ is the interior of $\left.\left[a_{i}, b_{i}\right]\right), \cup_{i \leq \nu}\left[a_{i}, b_{i}\right]=[-\pi, \pi]$ and $x_{i} \in\left(a_{i}, b_{i}\right)$ if $x_{i}$ is an interior point of $[-\pi, \pi]$. The points $\left\{x_{i}\right\}_{i \leq \nu}$ are the tags of $P[-\pi, \pi],[1,3]$. If $E$ is a subset of $[-\pi, \pi]$, then the restriction of $P[-\pi, \pi]$ to $E$ is a finite subset of $\left(\left[a_{i}, b_{i}\right], x_{i}\right) \in P[-\pi, \pi]$ such that each pair of sets $\left[a_{i}, b_{i}\right]$ and $E$ intersects in at least one point. In symbols, $\left.P[-\pi, \pi]\right|_{E}=\left\{\left(\left[a_{i}, b_{i}\right], x_{i}\right) \in P[-\pi, \pi] \mid\left[a_{i}, b_{i}\right] \cap E \neq \emptyset\right\}$. It is evident that a given partition of $[-\pi, \pi]$ can be tagged in infinitely many ways by choosing different points as tags. Given $\delta:[-\pi, \pi] \mapsto \mathbb{R}_{+}$, named a gauge, a partition $P[-\pi, \pi]$ is called $\delta$-fine if $\left[a_{i}, b_{i}\right] \subset\left(x_{i}-\delta\left(x_{i}\right), x_{i}+\delta\left(x_{i}\right)\right)$. Let $\mathcal{P}[-\pi, \pi]$ be the family of all partitions $P[-\pi, \pi]$ of $[-\pi, \pi]$. Then, by $\mathcal{P}_{\delta}[-\pi, \pi]$ we denote the family of all $\delta$-fine partitions $P[-\pi, \pi]$ of $[-\pi, \pi]$ for some given $\delta:[-\pi, \pi] \mapsto \mathbb{R}_{+}$.

For the infinite set of partitions $\left\{P_{n}[-\pi, \pi] \mid P_{n}[-\pi, \pi]=\left\{\left(\left[a_{i_{n}}, b_{i_{n}}\right], x_{i_{n}}\right)\right\}\right.$, $n \in \mathbb{N}\}$, denoted by $\left\langle P_{n}[-\pi, \pi]\right\rangle$, we write $\langle P[-\pi, \pi]\rangle \in(\mathcal{P}[-\pi, \pi], \prec)$, if $P_{n}[-\pi, \pi] \prec P_{n+1}[-\pi, \pi]$ for each $n \in \mathbb{N}$. The statement $P_{n}[-\pi, \pi] \prec$ $P_{n+1}[-\pi, \pi]$ means that for each interval-point pair $\left(\left[a_{i_{n+1}}, b_{i_{n+1}}\right], x_{i_{n+1}}\right) \in$ $P_{n+1}[-\pi, \pi]$ there exists a corresponding interval-point pair $\left(\left[a_{i_{n}}, b_{i_{n}}\right], x_{i_{n}}\right) \in$ $P_{n}[-\pi, \pi]$ such that $\left[a_{i_{n+1}}, b_{i_{n+1}}\right] \subset\left[a_{i_{n}}, b_{i_{n}}\right]$, and

$$
\begin{aligned}
\left\{x_{i_{n}} \mid\left(\left[a_{i_{n}}, b_{i_{n}}\right], x_{i_{n}}\right)\right. & \left.\in P_{n}[-\pi, \pi]\right\} \\
& \subset\left\{x_{i_{n+1}} \mid\left(\left[a_{i_{n+1}}, b_{i_{n+1}}\right], x_{i_{n+1}}\right) \in P_{n+1}[-\pi, \pi]\right\} .
\end{aligned}
$$

Clearly, for any $x \in[-\pi, \pi]$ there exists a directed set $\left\langle P_{n}[-\pi, \pi]\right\rangle \in$ $(\mathcal{P}[-\pi, \pi], \prec)$ so that $x$ is a tag for it.

In what follows we will use the following notations $\Delta F(I)=F(v)-F(u)$, where $u$ and $v$ are the endpoints of $I, \sum_{i_{n}} \Delta F\left(\left[a_{i_{n}}, b_{i_{n}}\right]\right)=\Delta F\left(P_{n}[-\pi, \pi]\right)$ and $\sum_{i_{n}} f\left(x_{i_{n}}\right)\left|\left[a_{i_{n}}, b_{i_{n}}\right]\right|=(f \Delta x)\left(P_{n}[-\pi, \pi]\right)$, whenever $\left(\left[a_{i_{n}}, b_{i_{n}}\right], x_{i_{n}}\right) \in$ $P_{n}[-\pi, \pi]$. In addition, if $t \in(0, \pi)$ and $\gamma(I, t)$ is an interval function associated to $\gamma(x, t)$, then

$$
\begin{aligned}
\sum_{i_{n}}\left[\gamma\left(\left[a_{i_{n}}, b_{i_{n}}\right], t\right) \Delta F\left(\left[a_{i_{n}}, b_{i_{n}}\right]\right)-\gamma\left(x_{i_{n}}, t\right)\left|\left[a_{i_{n}}, b_{i_{n}}\right]\right|\right] & \\
= & (\gamma \Delta F-\gamma \Delta x)\left(P_{n}[-\pi, \pi], t\right) .
\end{aligned}
$$

The following two definitions come from [2] and [5].

Definition 1. Let $f:[-\pi, \pi] \mapsto \mathbb{R}$. The point function $f$ is $\mathrm{H}_{1}$-integrable to a real point $\mathcal{A}$ on $[-\pi, \pi]$, if there exists a gauge $\delta$ on $[-\pi, \pi]$ such that for every $\varepsilon>0$ there exists a $\delta$-fine partition $P_{n_{\varepsilon}}[-\pi, \pi]$ such that

$$
\left|(f \Delta x)\left(P_{n}[-\pi, \pi]\right)-\mathcal{A}\right|<\varepsilon,
$$


whenever $P_{n}[-\pi, \pi] \in\left\langle P_{n}[-\pi, \pi]\right\rangle,\left\langle P_{n}[-\pi, \pi]\right\rangle \in\left(\mathcal{P}_{\delta}[-\pi, \pi], \prec\right)$ and $P_{n_{\varepsilon}}[-\pi, \pi] \prec P_{n}[-\pi, \pi]$. In symbols, $\mathcal{A}:=\mathrm{H}_{1}-\int_{-\pi}^{\pi} f d x$.

Definition 2. Let $\gamma: \mathcal{I}[-\pi, \pi] \mapsto \mathbb{R}$ be an arbitrary interval function. Then, a point function $g:[-\pi, \pi] \mapsto \mathbb{R}$ is the Moore-Smith limit of $\gamma$ on $E \subseteq[-\pi, \pi]$, if there exists a gauge $\delta$ on $[-\pi, \pi]$ such that for every $\varepsilon>0$ there exists a $\delta$-fine partition $P_{n_{\varepsilon}}[-\pi, \pi]$ such that

$$
\left|\gamma\left(\left[a_{i_{n}}, b_{i_{n}}\right]\right)-g\left(x_{i_{n}}\right)\right|<\varepsilon,
$$

whenever $\left.\left(\left[a_{i_{n}}, b_{i_{n}}\right], x_{i_{n}}\right) \in P_{n}[-\pi, \pi]\right|_{E}, P_{n}[-\pi, \pi] \in\left\langle P_{n}[-\pi, \pi]\right\rangle,\left\langle P_{n}\right.$ $[-\pi, \pi]\rangle \in\left(\mathcal{P}_{\delta}[-\pi, \pi], \prec\right)$ and $P_{n_{\varepsilon}}[-\pi, \pi] \prec P_{n}[-\pi, \pi]$.

For a primitive $F:[-\pi, \pi] \mapsto \mathbb{R}$, the derivative $f$ on $[-\pi, \pi]$ could be defined as the Moore-Smith limit of the interval function $\phi: \mathcal{I}[-\pi, \pi] \mapsto \mathbb{R}$ defined by

$$
\phi(I)=\frac{\Delta F(I)}{\Delta x(I)}=\frac{\Delta F}{\Delta x}(I),
$$

where $\Delta x(I)=|I|$. In this case, according to Definition 2 , given $\varepsilon>0$ there exists a gauge $\delta$ on $[-\pi, \pi]$ such that for every $\varepsilon>0$ there exists a $\delta$ fine partition $P_{n_{\varepsilon}}[-\pi, \pi]$ such that $\left|\Delta F\left(\left[a_{i_{n}}, b_{i_{n}}\right]\right)-f\left(x_{i_{n}}\right) \Delta x\left(\left[a_{i_{n}}, b_{i_{n}}\right]\right)\right| \leq$ $\varepsilon \Delta x\left(\left[a_{i_{n}}, b_{i_{n}}\right]\right)$, whenever $\left.\left(\left[a_{i_{n}}, b_{i_{n}}\right], x_{i_{n}}\right) \in P_{n}[-\pi, \pi]\right|_{E}, P_{n}[-\pi, \pi] \in\left\langle P_{n}\right.$ $[-\pi, \pi]\rangle,\left\langle P_{n}[-\pi, \pi]\right\rangle \in\left(\mathcal{P}_{\delta}[-\pi, \pi], \prec\right)$ and $P_{n_{\varepsilon}}[-\pi, \pi] \prec P_{n}[-\pi, \pi]$. Accordingly, if $E \subset[-\pi, \pi]$, more precisely if $F:[-\pi, \pi] \mapsto \mathbb{R}$ is a function that is not differentiable on $[-\pi, \pi]$, then for a given $\varepsilon>0$ in the set

$$
\Omega_{\varepsilon}^{\mathcal{K H}}=\{(x, I): x \in[-\pi, \pi] \text { is inside } I \text { and }|\Delta F(I)| \geq \varepsilon|I|\}
$$

we isolate two subsets:

$$
\begin{aligned}
& \Omega_{<\varepsilon}^{\mathcal{K H}}=\{(x, I): x \in[-\pi, \pi] \text { is inside } I \text { and } \varepsilon|I| \leq|\Delta F(I)|<\varepsilon\} \text { and } \\
& \Omega_{\geq \varepsilon}^{\mathcal{K H}}=\{(x, I): x \in[-\pi, \pi] \text { is inside } I \text { and }|\Delta F(I)| \geq \varepsilon\} .
\end{aligned}
$$

Definition 3. Let $F:[-\pi, \pi] \mapsto \mathbb{R}$. The set (vss) $[-\pi, \pi]=\{x \in[-\pi, \pi]$ : for every $\varepsilon>0$ there exists a $\delta$-fine $\left.(x, I) \in \Omega_{<\varepsilon}^{\mathcal{K H}}\right\}$ is said to be the set of apparent singular points of $F$ on $[-\pi, \pi]$.

Definition 4. Let $F:[-\pi, \pi] \mapsto \mathbb{R}$. The set $(v s)[-\pi, \pi]=\{x \in[-\pi, \pi]$ : for every $\varepsilon>0$ there exists a $\delta$-fine $\left.(x, I) \in \Omega_{\geq \varepsilon}^{\mathcal{K} \mathcal{H}}\right\}$ is said to be the set of singular points of $F$ on $[-\pi, \pi]$.

When working with functions, which have a finite number of discontinuities on $[-\pi, \pi]$, it does not really matter, from the point of view of totalization of the $\mathrm{H}_{1}$ integral, how these functions will be defined on the set $E$ of discontinuities. Hence, we adopt the convention that such functions are equal to 0 at all points at which they can take values $\pm \infty$ or not be defined at all. Accordingly, we may define point functions $F_{e x}:[-\pi, \pi] \mapsto \mathbb{R}$ and 
$f_{\text {ex }}:[-\pi, \pi] \mapsto \mathbb{R}$ by extending $F$ and its derivative $f$ from $[-\pi, \pi] \backslash E$ to $E$ by $F_{e x}(x)=0$ and $f_{e x}(x)=0$ for $x \in E$, so that

$$
\begin{aligned}
& F_{\text {ex }}(x)=\left\{\begin{array}{ll}
F(x), & \text { if } x \in[-\pi, \pi] \backslash E \\
0, & \text { if } x \in E
\end{array}\right. \text { and } \\
& f_{\text {ex }}(x)= \begin{cases}f(x), & \text { if } x \in[-\pi, \pi] \backslash E \\
0, & \text { if } x \in E\end{cases}
\end{aligned}
$$

The following two definitions come from [9].

Definition 5. Let $\gamma: \mathcal{I}[-\pi, \pi] \mapsto \mathbb{R}$ be an arbitrary interval function and for $F:[-\pi, \pi] \mapsto \mathbb{R}$ let $\phi: \mathcal{I}[-\pi, \pi] \mapsto \mathbb{R}$ be an interval function defined by (2.1), that converge, according to Definition 2, to $g(x)$ and $f(x)$, respectively, almost everywhere on $[-\pi, \pi]$. A point function $g(x)$ is totally $\mathrm{H}_{1}$-integrable, with respect to the differential form $d F(x)=f(x) d x$, to a real point $\mathcal{F}$ on $[-\pi, \pi]$ if there exists a gauge $\delta$ on $[-\pi, \pi]$ such that for every $\varepsilon>0$ there exists a $\delta$-fine partition $P_{n_{\varepsilon}}[-\pi, \pi]$ such that

$$
\left|(\gamma \Delta F)\left(P_{n}[-\pi, \pi]\right)-\mathcal{F}\right|<\varepsilon
$$

whenever $P_{n}[-\pi, \pi] \in\left\langle P_{n}[-\pi, \pi]\right\rangle,\left\langle P_{n}[-\pi, \pi]\right\rangle \in\left(\mathcal{P}_{\delta}[-\pi, \pi], \prec\right)$ and $P_{n_{\varepsilon}}$ $[-\pi, \pi] \prec P_{n}[-\pi, \pi]$. In symbols, $\mathcal{F}:=\mathrm{H}_{1}-v t \int_{-\pi}^{\pi} g d F$.

Remark 1. In case, any of the point functions $g$ and $f$ above is the MooreSmith limit of the corresponding interval function on $[-\pi, \pi]$, then in the previous definition (2.3) can be replaced by

$$
\left|(g \Delta F)\left(P_{n}[-\pi, \pi]\right)-\mathcal{F}\right|<\varepsilon \text { or }\left|(\gamma f \Delta x)\left(P_{n}[-\pi, \pi]\right)-\mathcal{F}\right|<\varepsilon,
$$

respectively.

Definition 6. Let $F:[-\pi, \pi] \mapsto \mathbb{R}$ and $E \subset(-\pi, \pi)$ be a set of Lebesgue measure zero such that $E=(v s)[-\pi, \pi]$. The linear differential form $d F(x)$ $=f(x) d x$, as the Moore-Smith limit of $\Delta F(I)=\phi(I) \Delta x(I)$ on $[-\pi, \pi]$, where $I \in \mathcal{I}([-\pi, \pi])$, is said to be basically summable $\left(\mathrm{BS}_{\delta}\right)$ to a real number $\Re$ on $E$ if there exists a gauge $\delta$ on $[-\pi, \pi]$ such that for every $\varepsilon>0$ there exists a $\delta$-fine partition $P_{n_{\varepsilon}}[-\pi, \pi]$ such that

$$
\left|\left(\Delta F-f_{e x} \Delta x\right)\left(\left.P_{n}[-\pi, \pi]\right|_{E}\right)-\Re\right|<\varepsilon,
$$

whenever $P_{n}[-\pi, \pi] \in\left\langle P_{n}[-\pi, \pi]\right\rangle,\left\langle P_{n}[-\pi, \pi]\right\rangle \in\left(\mathcal{P}_{\delta}[-\pi, \pi], \prec\right)$ and $P_{n_{\varepsilon}}$ $[-\pi, \pi] \prec P_{n}[-\pi, \pi]$. If in addition $E$ can be written as a countable union of sets on each of which the linear differential form $f(x) d x$ is $\mathrm{BS}_{\delta}$, then $f(x) d x$ is said to be $\mathrm{BSG}_{\delta}$ on $E$. In symbols, $\Re:=\sum_{x \in E} f(x) d x$. 


\section{Main Results}

It is an old result (see [9]) that if $\gamma:[-\pi, \pi] \mapsto \mathbb{R}$ is a point function defined by $\gamma(x, t)=\sum_{k=1}^{+\infty} \Gamma_{k}(x, t)+(x-t) / 2$, where $\Gamma_{k}(x, t)=\sin [k(x-t)] / k$, for every fixed $t \in(0, \pi)$, then the dispersion of function values on $[-\pi, \pi]$ is as follows

$$
\gamma(x, t)=\left\{\begin{array}{ll}
-\frac{\pi}{2}, & \text { if } x \in[-\pi, t) \\
0, & \text { if } x=t \\
\frac{\pi}{2}, & \text { if } x \in(t, \pi]
\end{array} .\right.
$$

Let $\gamma: \mathcal{I}([-\pi, \pi]) \mapsto \mathbb{R}$ be the associated interval function of $\gamma$. If $I \in$ $\mathcal{I}([-\pi, \pi])$ and $\gamma(I, t)=\gamma(v, t)-\gamma(u, t)$, where $u$ and $v$ are the endpoints of $I$, then

$$
\gamma(I, t)=\sum_{k=1}^{+\infty} \Gamma_{k}(I, t)+\frac{I}{2}= \begin{cases}\pi, & \text { if } t \in \text { int.I } \\ \frac{\pi}{2}, & \text { if } t \text { is the endpoint of } I \\ 0, & \text { if } t \notin I\end{cases}
$$

where $\Gamma_{k}(I, t)=\Gamma_{k}(v, t)-\Gamma_{k}(u, t)$. In addition, let $E \subset(-\pi, \pi)$ be a set of Lebesgue measure zero at whose points an arbitrary point function $F$, defined and differentiable to $f$ on $[-\pi, \pi] \backslash E$, can take values $\pm \infty$ or not be defined at all and $t \notin E$. If we introduce the analysis of the interval function $\gamma(I, t) \Delta F_{e x}(I)$, as the product of the two interval functions $\gamma(I, t)$ defind by (3.2) and $\Delta F_{e x}(I)$, whenever $I \in \mathcal{I}([-\pi, \pi])$, then, according to Definition 5,

$$
\mathrm{H}_{1}-v t \int_{-\pi}^{\pi}\left[\sum_{k=1}^{+\infty} G_{k}(x, t)+\frac{1}{2}\right] f(x) d x=\pi f(t),
$$

where $G_{k}(x, t)=\cos [k(x-t)]$ is the Moore-Smith limit of $\Gamma_{k}(I, t) / \Delta x(I)$, since $f(t)$ is the Moore-Smith limit of interval function $\phi(I)=\left(\Delta F_{\text {ex }} / \Delta x\right)$ $(I)$ at the point $t$ and therefore there exists a gauge $\delta$ on $[-\pi, \pi]$ such that for every $\varepsilon>0$ there exists a $\delta$-fine partition $P_{n_{\varepsilon}}[-\pi, \pi]$ such that

$$
\left|\left(\gamma \phi_{e x}\right)\left(P_{n}[-\pi, \pi], t\right)-\pi f(t)\right|=\pi\left|\phi_{e x}\left(\left[a_{i_{n}}, b_{i_{n}}\right]_{t}\right)-f(t)\right|<\pi \varepsilon,
$$

whenever $P_{n}[-\pi, \pi] \in\left\langle P_{n}[-\pi, \pi]\right\rangle,\left\langle P_{n}[-\pi, \pi]\right\rangle \in\left(\mathcal{P}_{\delta}[-\pi, \pi], \prec\right)$ and $P_{n_{\varepsilon}}$ $[-\pi, \pi] \prec P_{n}[-\pi, \pi]$, where $\left[a_{i_{n}}, b_{i_{n}}\right]_{t}$ are the subintervals $\left[a_{i_{n}}, b_{i_{n}}\right]$ to which the point $t$ belongs.

On the other hand, considering the fact that $\sum_{k=1}^{+\infty} \Gamma_{k}(I, t)$ converges on $[-\pi, \pi]$ it follows that $\sum_{k=1}^{+\infty}\left(\boldsymbol{\Gamma}_{k} / \Delta x\right)(P[-\pi, \pi], t)=\left(\sum_{k=1}^{+\infty} \boldsymbol{\Gamma}_{k} / \Delta x\right)(P$ $[-\pi, \pi], t)$, for every $P[-\pi, \pi] \in \mathcal{P}[-\pi, \pi]$. Hence, (3.3) becomes the Fourier 
series of $f$ at the point $t$, as follows

$$
\frac{1}{\pi} \sum_{k=1}^{+\infty} \mathrm{H}_{1}-v t \int_{-\pi}^{\pi} G_{k}(x, t) f(x) d x+\frac{1}{2 \pi} \mathrm{H}_{1}-v t \int_{-\pi}^{\pi} f(x) d x=f(t) .
$$

The following theorem gives us the opportunity to compute the Fourier coefficients for a function that can take not only finite but infinite values within $[-\pi, \pi]$, using the $\mathrm{H}_{1}$-integral, [9].

Theorem 1. For $[-\pi, \pi] \in \mathbb{R}$ let $E \subset(-\pi, \pi)$ be a set of Lebesgue measure zero such that a primitive $F$ is defined and differentiable on $[-\pi, \pi] \backslash E$ and its derivative $f$ can take values $\pm \infty$ or not be defined at all and let $t \in$ $(0, \pi) / E$. If $G_{k}(x, 0) d F(x)=G_{k}(x, 0) f(x) d x$, as the Moore-Smith limit of $G_{k}(x, 0) \Delta F_{\text {ex }}(I)$ on $[-\pi, \pi]$, where $I \in \mathcal{I}([-\pi, \pi])$, is basically summable $\left(B S_{\delta}\right)$ on $E$ to the sum $\Re_{k}$ and $G_{k}(x, 0) f_{e x}(x)$ is $H_{1}$-integrable to a real number $\mathcal{A}_{k}$ on $[-\pi, \pi]$, for each $k \in \mathbb{N}$, then

$$
H_{1}-v t \int_{-\pi}^{\pi} G_{k}(x, t) f(x) d x=H_{1}-\int_{-\pi}^{\pi} G_{k}(x, t) f(x) d x+\Re_{k} .
$$

Proof. Let $F_{e x}$ and $f_{e x}$ be defined by (2.3). Since the point function $G_{k}(x, 0)$ $f_{e x}(x)$ is $\mathrm{H}_{1}$-integrable to a real number $\mathcal{A}_{k}$ on $[-\pi, \pi]$ and $G_{k}(x, 0) d F(x)$ is $\left(\mathrm{BS}_{\delta}\right)$ on $E$ to $\Re_{k}$, for each $k \in \mathbb{N}$, it follows from Definitions 2 and 6 that there exists a gauge $\delta_{1}$ on $[-\pi, \pi]$ such that for every $\varepsilon>0$ there exists a $\delta_{1}$-fine partition $P_{n_{\varepsilon}}[-\pi, \pi]$ such that

$$
\left|\left(G_{k} f_{e x} \Delta x\right)\left(P_{n}[-\pi, \pi], 0\right)-\mathcal{A}_{k}\right|<\varepsilon,
$$

whenever $P_{n}[-\pi, \pi] \in\left\langle P_{n}[-\pi, \pi]\right\rangle,\left\langle P_{n}[-\pi, \pi]\right\rangle \in\left(\mathcal{P}_{\delta_{1}}[-\pi, \pi], \prec\right)$ and $P_{n_{\varepsilon}}$ $[-\pi, \pi] \prec P_{n}[-\pi, \pi]$, as well as a gauge $\delta_{2}$ on $[-\pi, \pi]$ such that for every $\varepsilon>0$ there exists a $\delta_{2}$-fine partition $P_{n_{\varepsilon}}[-\pi, \pi]$ such that

$$
\left|\left(G_{k} \Delta F_{e x}-G_{k} f_{e x} \Delta x\right)\left(\left.P_{n}[-\pi, \pi]\right|_{E}, 0\right)-\Re_{k}\right|<\varepsilon,
$$

whenever $P_{n}[-\pi, \pi] \in\left\langle P_{n}[-\pi, \pi]\right\rangle,\left\langle P_{n}[-\pi, \pi]\right\rangle \in\left(\mathcal{P}_{\delta_{2}}[-\pi, \pi], \prec\right)$ and $P_{n_{\varepsilon}}$ $[-\pi, \pi] \prec P_{n}[-\pi, \pi]$. In addition $f_{e x}(x) \equiv 0$ on $E$ and $G_{k}(x, 0) d F(x)$ is the Moore-Smith limit of $G_{k}(x, 0) \Delta F(I)$ on $[-\pi, \pi] \backslash E$, that means that there exits a gauge $\delta_{3}$ on $[-\pi, \pi]$ such that for every $\varepsilon>0$ there exists a $\delta_{2}$-fine partition $P_{n_{\varepsilon}}[-\pi, \pi]$ such that

$$
\left|\left(G_{k} \Delta F-G_{k} f \Delta x\right)\left(\left.P_{n}[-\pi, \pi] \backslash P_{n}[-\pi, \pi]\right|_{E}, 0\right)\right| \leq 2 \pi \varepsilon,
$$

whenever $P_{n}[-\pi, \pi] \in\left\langle P_{n}[-\pi, \pi]\right\rangle,\left\langle P_{n}[-\pi, \pi]\right\rangle \in\left(\mathcal{P}_{\delta_{3}}[-\pi, \pi], \prec\right)$ and $P_{n_{\varepsilon}}$ $[-\pi, \pi] \prec P_{n}[-\pi, \pi]$. A gauge $\delta$ may be chosen so that $\delta(x)=\min \left(\delta_{1}(x)\right.$, $\left.\delta_{2}(x), \delta_{3}(x)\right)$ on $[-\pi, \pi]$. Hence, there exists a gauge $\delta$ on $[-\pi, \pi]$ such that 
for every $\varepsilon>0$ there exists a $\delta$-fine partition $P_{n_{\varepsilon}}[-\pi, \pi]$ such that

$$
\begin{gathered}
\left|\left(G_{k} \Delta F_{e x}\right)\left(P_{n}[-\pi, \pi], 0\right)-\mathcal{A}_{k}-\Re_{k}\right| \leq\left|\left(G_{k} f_{e x} \Delta x\right)\left(P_{n}[-\pi, \pi], 0\right)-\mathcal{A}_{k}\right| \\
+\left|\left(G_{k} \Delta F_{e x}-G_{k} f_{e x} \Delta x\right)\left(P_{n}[-\pi, \pi], 0\right)-\Re_{k}\right|
\end{gathered}
$$

that is,

$$
\begin{aligned}
\mid\left(G_{k} \Delta F_{e x}\right. & \left.-G_{k} f_{e x} \Delta x\right)\left(P_{n}[-\pi, \pi], 0\right)-\Re_{k} \mid \\
& \leq\left|\left(G_{k} \Delta F-G_{k} f \Delta x\right)\left(\left.P_{n}[-\pi, \pi] \backslash P_{n}[-\pi, \pi]\right|_{E}, 0\right)\right| \\
& +\left|\left(G_{k} \Delta F_{e x}-G_{k} f_{e x} \Delta x\right)\left(\left.P_{n}[-\pi, \pi]\right|_{E}, 0\right)-\Re_{k}\right| \leq(2 \pi+1) \varepsilon,
\end{aligned}
$$

whenever $P_{n}[-\pi, \pi] \in\left\langle P_{n}[-\pi, \pi]\right\rangle,\left\langle P_{n}[-\pi, \pi]\right\rangle \in\left(\mathcal{P}_{\delta}[-\pi, \pi], \prec\right)$ and $P_{n_{\varepsilon}}$ $[-\pi, \pi] \prec P_{n}[-\pi, \pi]$, so that there exists a gauge $\delta$ on $[-\pi, \pi]$ such that for every $\varepsilon>0$ there exists a $\delta$-fine partition $P_{n_{\varepsilon}}[-\pi, \pi]$ such that

$$
\left|\left(G_{k} \Delta F_{e x}\right)\left(P_{n}[-\pi, \pi], 0\right)-\mathcal{A}_{k}-\Re_{k}\right| \leq 2(1+\pi) \varepsilon,
$$

whenever $P_{n}[-\pi, \pi] \in\left\langle P_{n}[-\pi, \pi]\right\rangle,\left\langle P_{n}[-\pi, \pi]\right\rangle \in\left(\mathcal{P}_{\delta}[-\pi, \pi], \prec\right)$ and $P_{n_{\varepsilon}}$ $[-\pi, \pi] \prec P_{n}[-\pi, \pi]$. Therefore,

$$
\mathrm{H}_{1}-v t \int_{-\pi}^{\pi} G_{k}(x, 0) f(x) d x=\mathrm{H}_{1}-\int_{-\pi}^{\pi} G_{k}(x, 0) f_{e x}(x) d x+\Re_{k} .
$$

If $H(x)$ is the Heaviside (unit) step function, then $d H(x)=\delta(x) d x$, where $\delta(x)$ is the Dirac delta function that is zero everywhere except at zero, is the Moore-Smith limit of the interval function $\Delta H(I)$, associated to $H(x)$, on $[-\pi, \pi] \backslash E_{0}$, where $E_{0}=\{0\}$. Since there exists a gauge $\delta$ on $[-\pi, \pi]$ such that for every $\varepsilon>0$ there exists a $\delta$-fine partition $P_{n_{\varepsilon}}[-\pi, \pi]$ such that $\left|\Delta H\left(P_{n}[-\pi, \pi]\right)-1\right|<\varepsilon$ and $\left|\left(G_{k} \Delta H\right)\left(P_{n}[-\pi, \pi], 0\right)-1\right|<\varepsilon$, whenever $P_{n}[-\pi, \pi] \in\left\langle P_{n}[-\pi, \pi]\right\rangle,\left\langle P_{n}[-\pi, \pi]\right\rangle \in\left(\mathcal{P}_{\delta_{3}}[-\pi, \pi], \prec\right), P_{n_{\varepsilon}}[-\pi, \pi] \prec$ $P_{n}[-\pi, \pi]$ and $k \in \mathbb{N}$, it follows from Definition 5 that

$$
\mathrm{H}_{1}-v t \int_{-\pi}^{\pi} G_{k}(x, 0) d H(x)=1,
$$

for each $k \in \mathbb{N}_{0}$, where $\mathbb{N}_{0}=\mathbb{N} \cup\{0\}$.

By Theorem 1 and Definition 6 , we see that $\mathrm{H}_{1}-\int_{-\pi}^{\pi} G_{k}(x, 0) \delta_{e x}(x) d x \equiv$ 0 , for each $k \in \mathbb{N}_{0}$, since $G_{k}(x, 0) d H(x)$ are basically summable $\left(\mathrm{BS}_{\delta}\right)$ to 1 on $E_{0}$, for each $k \in \mathbb{N}_{0}$. Finally, it follows from (3.5) that

$$
\sum_{k=1}^{+\infty} G_{k}(0, t)+\frac{1}{2}=\pi \delta(t),
$$

at every point $t$ belonging to the set $[-\pi, \pi] \backslash E_{0}$. This confirms that $\sum_{k=1}^{+\infty} G_{k}$ $(x, t)$ is the Moore-Smith limit of $\sum_{k=1}^{+\infty} \Gamma_{k}(I, t) / \Delta x(I)$ on $[-\pi, \pi] \backslash E_{t}$, where 
$E_{t}=\{t\}$ and $t \in(-\pi, \pi)$. In addition, for any real-valued periodic function $f(x)$ of period $2 \pi$, which is defined at a point $t \in(-\pi, \pi)$, it follows from (3.5) that

$$
\begin{aligned}
\pi f(t)=\mathrm{H}_{1}-v t \int_{-\pi}^{\pi} \pi \delta(x-t) f(x) d x & \\
=\mathrm{H}_{1}-v t \int_{-\pi}^{\pi}\left[\sum_{k=1}^{+\infty} G_{k}(x, t)+\frac{1}{2}\right] f(x) d x= & \sum_{k=1}^{+\infty} \mathrm{H}_{1}-v t \int_{-\pi}^{\pi} G_{k}(x, t) f(x) d x \\
& +\frac{1}{2} \mathrm{H}_{1}-v t \int_{-\pi}^{\pi} f(x) d x
\end{aligned}
$$

\section{REFERENCES}

[1] R. G. Bartle, A Modern Theory of Integration, Graduate Studies in Math., Vol. 32, AMS, Providence, 2001.

[2] I. J. L. Garces and P. Y. Lee, Convergence theorems for the $H_{1}$-integral, Taiwanese J. Math., 4 (3) (2000), 439-445.

[3] R. A. Gordon, The Integrals of Lebesgue, Denjoy, Perron and Henstock, Graduate Studies in Math., Vol. 4, AMS, Providence, 1994.

[4] J. Marcinkiewicz and A. Zygmund, Two theorems on trigonometrical series, Rec. Math., [Mat. Sbornik] N.S., 2 (44) 4, (1937), 733-737.

[5] E. J. McShane, Partial orderings and Moore-Smith limits, Am. Math. Mon., 59 (1952), $1-11$.

[6] B. Sarić, The Fourier series of one class of functions with discontinuities, Doctoral dissertation defended on 20th of October 2009 at the University of Novi Sad, Faculty of Science, Department of Mathematics and Informathics.

[7] B. Sarić, Cauchy's residue theorem for a class of real valued functions, Czech. Math. J., 60 (4) (2010), 1043-1048.

[8] B. Sarić, On totalization of the Henstock - Kurzweil integral in the multidimensional space, Czech. Math. J., 61, (4) (2011), 1017-1022.

[9] B. Sarić, On totalization of the $H_{1}$-integral, Taiw. J. Math., 15 (4) (2011), 1691-1700.

[10] V. Sinha and I. K. Rana, On the continuity of associated interval functions, Real Anal. Exch., 29 (2) (2003/2004), 979-981.

[11] A. Zygmund, Trigonometric series, University Press, Cambridge, 2003.

(Received: March 30, 2013)

(Revised: July 30, 2013)
Faculty of Sciences University of Novi Sad Trg Dositeja Obradovića 2 21000 Novi Sad, Serbia College of Technical Engineering Professional Studies Svetog Save 65 32000 Čačak, Serbia saric.b@open.telekom.rs 